\title{
Observational Study Protocol Version
}

National Cancer Institute

\section{Source}

National Cancer Institute. Observational Study Protocol Version. NCI Thesaurus. Code C93487.

An action plan of a pre-clinical or clinical study in which biomedical and/or health outcomes are assessed in pre-defined groups of individuals. Study subjects in the study may receive diagnostic, therapeutic, or other interventions, but the investigator does not assign specific interventions to the study subjects or experimental units of the study. 